\title{
Sur les assemblages de lignes.
}

(Par M. Camille Jordan à Paris.)

1. Soient $x, y, z, u, \ldots$ des points en nombre quelconque; $x y, x z$, $y u, \ldots$ des arêtes droites ou courbes, mais non bifurquées, dont chacune joint ensemble deux de ces points. Nous appellerons un semblable système un assemblage d'arêtes, dont $x, y, z, u, \ldots$ sont les sommets. Un tel assemblage peut être représenté symboliquement par la forme quadratique $x y+x z+y u+\cdots$, dont les divers termes représentent les diverses arêtes de l'assemblage. Si d'ailleurs $a$ de ces arêtes ont les mêmes extrémités $x$, $y$, la forme représentative de l'assemblage contiendra $a$ fois le terme $x y$.

Soient maintenant $x y, x z, \ldots$ ceux des termes de la forme quadratique qui contiennent $x ; y u, \ldots$ ceux qui ont une lettre commune avec quelqu'un de ceux-là; $u v, \ldots$ ceux qui ont une lettre commune avec les précédents, etc. ... Il est clair que l'assemblage sera continu si l'ensemble de ces termes $x y, x z, \ldots, y u, \ldots, u v, \ldots$ contient toutes les lettres: sinon, non. L'assemblage sera dit $n+1$ fois continu s'il est possible de couper $n$ des arêtes qui le forment sans détruire sa continuité.

Deux assemblages $\boldsymbol{A}$ et $\boldsymbol{A}^{\prime}$ seront dits pareils, si l'on peut établir entre leurs arêtes et sommets une correspondance telle, qu'à chaque arête $x y$ de l'un des assemblages corresponde dans l'autre une arête $x^{\prime} y^{\prime}$ joignant ensemble les sommets $x^{\prime}, y^{\prime}$ respectivement correspondants à $x$ et à $y$. Dans ce cas, si $a x y+b x z+\cdots$ est la forme représentative de $A, a x^{\prime} y^{\prime}+b x^{\prime} z^{\prime}+\cdots$ sera évidemment la forme représentative de $A^{\prime}$.

Un assemblage peut être pareil à lui-même sous divers points de vue. Dans ce cas, $A^{\prime}$ se confondra avec $A$, et $x^{\prime}, y^{\prime}, z^{\prime}, \ldots$ seront, à l'ordre près, identiques à $x, y, z, \ldots$ De plus les deux formes $a x y+b x z+\cdots$ et $a x^{\prime} y^{\prime}+b x^{\prime} z^{\prime}+\cdots$, représentatives du même réseau, seront nécessairement identiques. Donc la substitution qui remplace $x, y, z, \ldots$ par $x^{\prime}, y^{\prime}, z^{\prime}, \ldots$ n'altère pas la forme $a x y+b x z+\cdots$ Réciproquement, soit $S$ une substitution qui n'altère pas cette forme, et supposons qu'elle remplace respectivement $x$, $y, z, \ldots$ par $x^{\prime}, y^{\prime}, z^{\prime}, \ldots$ Faisons correspondre aux sommets $x, y, z, \ldots$ les sommets $x^{\prime}, y^{\prime}, z^{\prime}, \ldots$; aux $a$ arêtes qui joignent $x$ et $y$ les arêtes analogues qui joignent les sommets analogues $x^{\prime}, y^{\prime}$, ces dernières arêtes étant 
prises dans un ordre quelconque; etc. ...; l'assemblage restera pareil à lui-même.

L'ordre de symétrie de l'assemblage, c'est-à - dire le nombre de manières différentes dont il est pareil à lui -même est donc égal à $1.2 \ldots a .1 .2 \ldots b \ldots N$, $N$ étant le nombre des substitutions telles que $S$.

2. Problème. Quels sont les assemblages continus, non pareils entre eux, que l'on peut former avec $m$ arêtes? Quels sont le degré de continuité et la symétrie de chacun d'eux?

Le tableau ci-joint donne la solution de ce problème pour $m=2,3$ ou 4. Chaque assemblage y est désigné par sa forme représentative. ( $0 \mathrm{n}$ construira aisément la figure géométrique correspondante.) En regard sont indiqués son degré de continuité (en chiffres romains) et son ordre de symétrie (en chiffres arabes).

$$
\begin{aligned}
& m=2\{a b+b c \quad \text { I; } 2 \mid 2 a b \quad \text { II } ; 4 \\
& m=3\left\{\begin{array}{lr|lr}
a b+b c+c d & \text { I; } 2 & 2 a b+b c & \text { II } ; 2 \\
a b+b c+b d & \text { I; } 6 & 3 a b & \text { III } 12 \\
a b+b c+c a & \text { II } 6 & &
\end{array}\right.
\end{aligned}
$$

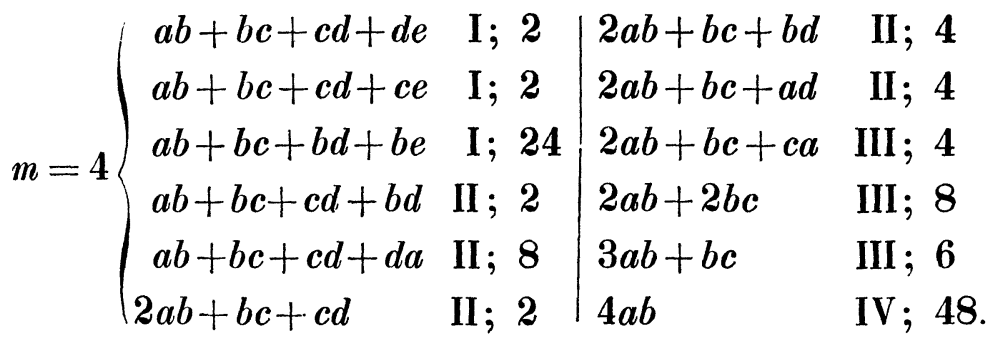

On étendrait sans peine ce tableau au cas où $m=5$, etc .... Mais la complication croîtrait assez vite. On obtient plus aisément des résultats ayant quelque généralité, en laissant $m$ indéterminé, et examinant successivement les assemblages à continuité simple, double, triple, etc. . . .

3. Assemblages à continuité simple. Soit $x$ un sommet quelconque de l'assemblage. On peut distinguer dans l'assemblage diverses branches, formées chacune par l'une des arêtes $x y_{1}, x y_{2}, \ldots$ qui aboutissent au sommet $x$ et par l'ensemble des arêtes qui se rattachent à $x$ par l'intermédiaire de celle-là. Soient $p_{1}, p_{2}, \ldots$ les nombres d'arêtes respectivement contenues dans ces diverses branches; $q$ le plus grand de ces nombres. L'assemblage contiendra un sommet pour lequel $q \overline{\bar{\chi}} \frac{m}{2}$, ou deux sommets, situés aux deux extrémités 
de la même arête et pour lesquels $q=\frac{m+1}{2}$. Pour tous les autres sommets de l'assemblage, $q$ sera $>\frac{m+1}{2}$.

Supposons en effet que $x$ soit choisi de telle sorte que $q$ atteigne son minimum, et soit pour fixer les idées $q_{1}=q$. Soient $x y_{1}, x^{\prime} y_{1}, x^{\prime \prime} y_{1}, \ldots$ les arêtes qui aboutissent au sommet $y_{1} ; r, r^{\prime}, r^{\prime \prime}, \ldots$ les nombres respectifs d'arêtes qui se rattachent à $y_{1}$ par l'intermédiaire de ces arêtes: on aura évidemment $r=m-q+1, r^{\prime}+r^{\prime \prime}+\cdots=q-1$. Donc $q \equiv \frac{m+1}{2}$, sans quoi on aurait à la fois $r, r^{\prime}, r^{\prime \prime}, \ldots<q$, contrairement à l'hypothèse.

Cela posé, soit d'abord $q<\frac{m+1}{2}$. Soient $u$ un sommet quelconque autre que $x ; x y_{\mu}, \ldots, z u$ les arètes successives, en nombre $k$, par lesquelles il se rattache à $x$. Le nombre des arêtes qui se rattachent à $u$ par l'intermédiaire de l'arête $z u$ est évidemment égal ou supérieur à $k+m-p_{\mu}$, nombre supérieur à $\frac{m+1}{2}, k$ étant au moins égal à 1 et $p_{\mu}$ au plus égal à $q$.

Si $q=\frac{m+1}{2}$ la même démonstration s'applique, à moins que l'on n'ait à la fois $k=1$ et $p_{\mu}=q=\frac{m+1}{2}$, d'où $\mu=1$, auquel cas le sommet $u$ se réduit à $y_{1}$.

On peut appeler centre de l'assemblage le sommet ou l'arête remarquable dont nous venons d'établir l'existence. Il est clair que ce centre se correspond à lui-même sous tous les aspects relativement auxquels l'assemblage serait pareil à lui-même.

Considérons maintenant un assemblage quelconque: admettons, pour fixer les idées, que son centre soit un sommet, et que les branches issues de ce point se répartissent ainsi: $1^{\circ} k$ branches formées de $n$ arêtes, parmi lesquelles il en existe $k_{1}$ pareilles entre elles (c'est à dire où les arêtes soient assemblées dans le même ordre); puis $k_{2}$ pareilles entre elles et différentes des précédentes; etc. $2^{\circ} k^{\prime}$ branches formées de $n^{\prime}$ arêtes, parmi lesquelles il en existe $k_{1}^{\prime}$ pareilles entre elles, puis $k_{2}^{\prime}$ pareilles entre elles et différentes des précédentes, etc. $\ldots 3^{\circ}$ etc. ... Supposons en outre que parmi les nombres $k_{1}, k_{2}, \ldots$ il y en ait $a_{1}$ égaux entre eux, puis $a_{2}$ égaux entre eux mais différents des précédents, etc....; que parmi les nombres $k_{1}^{\prime}, k_{2}^{\prime}, \ldots$ il y en ait $a_{1}^{\prime}$ égaux entre eux, puis $a_{2}^{\prime}$ égaux entre eux et différents des précédents, etc.... Soient $\delta$ le nombre des quantités $a_{1}, a_{2}, \ldots, \delta^{\prime}$ le nombre des quantités $a_{1}^{\prime}, a_{2}^{\prime}, \ldots$ etc..... Soit enfin $B_{n}$ le nombre de manières diffèrentes dont on peut con24 * 
stituer une branche avec $n$ lignes. Il est clair que le nombre des assemblages différents correspondants aux valeurs données de $n, k, k_{1}, k_{2}, \ldots, n^{\prime}, k^{\prime}, k_{1}^{\prime}$, $k_{2}^{\prime}, \ldots$ sera

(1.) $\frac{B_{n}\left(B_{n}-1\right) \ldots\left(B_{n}-\delta+1\right)}{1.2 \ldots a_{1} \cdot 1.2 \ldots a_{2} \ldots 1.2 \ldots a_{\delta}} \cdot \frac{B_{n^{\prime}}\left(B_{n^{\prime}}-1\right) \ldots\left(B_{n^{\prime}}-\delta^{\prime}+1\right)}{1.2 \ldots a_{1}^{\prime} 1.2 \ldots a_{2}^{\prime} \ldots 1.2 \ldots a_{\delta^{\prime}}^{\prime}} \cdots$

et l'on aura le nombre total des assemblages formés avec $m$ arêtes en sommant les expressions relatives à tous les systèmes de valeurs de ces quantités qui satisfont aux relations

$$
\begin{gathered}
k n+k^{\prime} n^{\prime}+\cdots=m, \quad k_{1}+k_{2}+\cdots=k, \quad k_{1}^{\prime}+k_{2}^{\prime}+\cdots=k^{\prime}, \ldots \\
\text { (3.) } \quad \frac{m+1}{2}>n>n^{\prime} \ldots
\end{gathered}
$$

dont la dernière exprime que $x$ est le centre de l'assemblage.

L'ordre de symétrie de chacun des assemblages ainsi obtenus sera évidemment égal à

$$
1.2 \ldots k_{1} S_{1}^{k_{1}} .1 .2 \ldots k_{2} S_{2}^{k_{2}} \ldots 1.2 \ldots k_{1}^{\prime} S_{1}^{\prime k_{1}^{\prime}} .1 .2 \ldots k_{2}^{\prime} S_{2}^{\prime k_{2}^{\prime}} \ldots
$$

$S_{1}, S_{2}, \ldots, S_{1}^{\prime}, S_{2}^{\prime}, \ldots$ étant les ordres de symétrie respectifs des diverses sortes de branches qu'il contient.

Il reste à déterminer $B_{n}, S_{1}$, et les quantitès analogues. Remarquons pour cela qu'une branche formée de $n$ arêtes se compose $1^{\circ}$ d'une première arête $x y, 2^{\circ}$ d'autres arêtes en nombre $n-1$, formant ensemble un assemblage dont $y$ est un sommet: $B_{n}$ est évidemment égal au nombre de manières de construire cet assemblage, et $S_{1}$ à son ordre de symétrie. On obtiendra donc ces deux nombres par des formules analogues à celles ci-dessus, où $m$ serait remplacé par $n-1$. La sommation doit d'ailleurs être étendue à toutes les valeurs des variables qui satisfont aux relations analogues à (2.), car le point $y$ pouvant être un sommet quelconque du nouvel assemblage, on n'a ici aucune relation analogue à (3.).

Le cas des assemblages à arête centrale se traiterait non moins facilement.

4. Soit $A$ un assemblage quelconque à continuité simple. Parmi les arêtes qui le forment, il en existe nécessairement qui ne se rattachent au reste de l'assemblage que par une de leurs extrémités, l'autre restant libre. Supprimons-les toutes à la fois; supprimons de même dans l'assemblage restant $A^{\prime}$ toutes les arêtes qui ont une extrémité libre; et répétons cette opération jusqu'au moment où on ne peut plus la répéter sans faire disparaître toutes les arêtes restantes. Deux cas pourront alors se présenter: 


\section{$1^{\circ}$. Il ne reste plus qu'une arête.}

$2^{\circ}$. Il reste plusieurs arêtes, ayant toutes une extrémité libre. Leurs autres extrémités concourent évidemment en un même sommet.

Voilà donc une nouvelle manière de discerner dans l'assemblage une arête ou sommet remarquable, jouissant de propriétés à lui spéciales, et dont la position dans l'assemblage peut être considérée à certains égards comme centrale. Nous lui donnerons le nom de centre de seconde espèce.

5. On peut déterminer encore comme il suit deux autres sommets ou arêtes remarquables:

Nous distinguerons dans $A$ trois sortes de sommets: $1^{\circ}$ les sommets ordinaires, où aboutissent deux arêtes, $2^{\circ}$ les sommets terminaux, où il n'en aboutit qu'une, $3^{\circ}$ ceux de branchement, où il en aboutit plus de deux. Cela posé, l'assemblage peut évidemment être divisé en sections, limitées à des sommets terminaux ou de branchement. Chacune de ces sections sera d'ailleurs formée d'une seule arête, ou d'arêtes successives se joignant en des sommets ordinaires.

Ces diverses sections, considérées chacune comme un seul tout, forment un assemblage, où l'on peut déterminer par les méthodes précédentes deux centres, dont chacun correspondra à lui-même sous tous les aspects pour lesquels $A$ est pareil à lui-même. Le centre considéré peut être un sommet, ou une section. Dans le second cas, suivant que la section sera composée d'un nombre pair ou impair d'arêtes, son milieu sera un sommet ou une arête remarquable, lequel sera évidemment homologue à lui-même sous tous les aspects pour lesquels $\boldsymbol{A}$ est pareil à lui-même.

6. Assemblages à continuité double. Opérons comme au No.4. tant qu'il restera dans l'assemblage des arêtes ayant une extrémitẻ libre. Les lignes restantes formeront un contour fermé, toujours homologue à lui-même. Cette observation faite, on posera sans peine, comme au No. 3., les formules qui donnent la solution du problème.

7. Assemblages à continuité triple. Opérons comme au No. 4. Les arêtes restantes formeront un système $A^{\prime}$, triplement continu. On y pourra donc déterminer des arêtes formant un contour fermé $C$. Coupons l'une de ces arêtes: il restera un assemblage à continuité double, sur lequel on pourra répéter les opérations du No. 4. jusqu'à ce qu'il ne reste plus qu'un contour fermé $D$. Deux cas seront ici à distinguer. 
$1^{\circ}$. Si $C$ et $D$ n'ont aucune arête commune, $A^{\prime}$ sera formé de ces deux contours et d'une ligne de jonction $L$, généralement polygonale, mais qui pourra, dans certains cas particuliers, se réduire à une seule arête, ou même à un point. Le milieu de $L$ sera un centre de l'assemblage.

$2^{\circ}$. Si les contours $C$ et $D$ ont une portion commune, cette portion et les deux autres portions non communes forment un système de trois lignes ayant leurs extrémités en deux mêmes points, $p, p^{\prime}$, qu'on pourra appeler les pôles de l'assemblage: et il est clair que le système formé par ces deux points sera constamment homologue à lui-mème.

8. On continuera de même pour les assemblages à continuité quadruple, etc. ... Mais voici un résultat relatif aux assemblages quelconques.

Soient $\boldsymbol{A}$ un assemblage, $\boldsymbol{S}$ l'un de ses sommets. Partageons les arêtes qui aboutissent en $S$ en deux catégories; puis supposons qu'on disjoigne les arêtes de la première catégorie de celles de la seconde, sans rompre la liaison existante entre les arêtes d'une même catégorie. On peut appeler cette opération une scission. Et un assemblage sera composé ou primitif, suivant qu'on pourra ou non détruire sa continuité par une scission.

Cela posé, il est clair que tout assemblage composé pourra être fractionné par une suite de scissions convenables en assemblages partiels qui seront primitifs; et ces fragments élant supposés ressoudés entre eux, on voit aisément par des raisonnements analogues à ceux des No. 3, 4, 5, qu'il existe quatre manières différentes de déterminer dans l'assemblage un sommet ou fragment central, constamment homologue à lui-même.

Le problème de la symétrie des assemblages se trouve ainsi ramené au cas des assemblages primitifs.

Paris, 1869. 